\title{
Descriptive spatial epidemiology of subclinical Salmonella infection in finisher pig herds: application of a novel method of spatially adaptive smoothing
}

\author{
Jackie Benschop ${ }^{1 *}$, Martin L. HAZELton ${ }^{2}$, Mark A. SteVEnson ${ }^{1}$, Jan DAHL ${ }^{3}$, \\ Roger S. MORRIS ${ }^{1}$, Nigel P. FRENCH ${ }^{1}$ \\ ${ }^{1}$ EpiCentre, Institute of Veterinary, Animal, and Biomedical Sciences, Massey University, Private Bag 11-222, \\ Palmerston North, New Zealand \\ ${ }^{2}$ Institute of Information Sciences and Technology, Massey University, Private Bag 11-222, Palmerston North, \\ New Zealand \\ ${ }^{3}$ Danish Meat Association, Axeltorv 3, 1609 Copenhagen V, Denmark
}

(Received 22 March 2007; accepted 29 June 2007)

\begin{abstract}
We describe the spatial epidemiological features of the 6.8 million meat-juice serological tests that were conducted between 1995 and 2004 as part of the Danish swine Salmonella control programme. We investigated pig and farm density using edge-corrected kernel estimations. Pigs were aggregated at the county level to assess county-level risk, and then we investigated farm-level risk by giving farms a case or non-case label using a cut-off of $40 \%$ of pigs positive. Conditional probability surfaces, correcting for the underlying population at risk, were produced for each year of the study period using a novel kernel estimator with a spatially adaptive smoothing bandwidth. This approach improves on previous methods by allowing focussed estimation of risk in areas of high population density while maintaining stable estimates in regions where the data are sparse. Two spatial trends in the conditional probability of a farm being a case were evident: (1) over the whole country, with the highest risk in the west compared to the east; and (2) on the Jutland peninsula with the highest risk in the north and south. At the farm-level a consistent area of risk was the south-west of Jutland. Case farms tended to aggregate indicating spatial dependency in the data. We found no association between pig or farm density and Salmonella risk. We generated hypotheses for this spatial pattern of risk and we conclude that this spatial pattern should be considered in the development of surveillance strategies and as a basis for further, more detailed analyses of the data.
\end{abstract}

pigs / Salmonella / spatial epidemiology / Denmark / kernel smoothing

\section{INTRODUCTION}

Exploratory data analysis is the cornerstone of sound epidemiological investigation [27]. In exploratory spatial data analysis we aim to describe spatial variation in disease without any explicit attempt to represent this variation in terms of a probability model. Suitable tools include point maps, kernel smoothing and mapping of relative risk and conditional probability surfaces. These allow us to

*Corresponding author: j.benschop@massey.ac.nz generate hypotheses that give insight as to why disease is abundant in some areas but not in others.

Our motivation was to explore data from the Danish Swine Salmonellosis Control Programme (D.S.S.C.P.) established by the Danish Ministry of Food, Agriculture and Fisheries in 1993 [21]. The D.S.S.C.P. was set up in response to a general increase in the incidence of confirmed cases of human salmonellosis due to pork consumption [3] and a large, common source outbreak caused by 
Salmonella infantis, traced back to one slaughter plant and a small number of supplier pig herds [30]. The objective of the D.S.S.C.P. is to reduce the prevalence of Salmonella to an acceptably low level so that domestically produced pork is no longer an important source of human salmonellosis. At the time of writing this, the objective has largely been achieved: the number of cases of salmonellosis in humans attributable to pork consumption has reduced from 1444 in 1993 to 142 in $2004^{1}$ [25]. At present, the focus of the D.S.S.C.P. is increasingly on the efficiency of Salmonella surveillance, with both industry and authorities wanting to achieve the greatest reduction in the prevalence of Salmonella for their money.

In the light of this focus on efficiency, we used a novel kernel estimator with a spatially adaptive smoothing bandwidth to produce estimates of the conditional probability of Salmonella risk across Denmark. Identified areas with high probability of risk could be targeted for increased surveillance, while those with a low probability could be less frequently surveyed. This "risk-based" approach to surveillance would complement the recent strategy initiated in July 2005 where herds with a negative history of Salmonella are sampled less frequently ${ }^{2}$.

Earlier spatial analyses of data from the D.S.S.C.P. have described the geographical distribution of sero-prevalence [21], and fitted county of origin as a fixed effect in regression models that aimed to quantify the effect of factors that influence Salmonella sero-prevalence [9]. These studies have only considered data from 1995 from the D.S.S.C.P. while the analyses presented here consider data from 1995 to 2004. The identification of spatial patterns in the data will inform further, more detailed analyses as well as highlight areas with abundant Salmonella infection for targeted surveillance.

\footnotetext{
${ }^{1}$ Ministry of Family and Consumer Affairs, Annual Report on Zoonoses in Denmark 2004, 2005 , Copenhagen.

${ }^{2}$ Ministry of Family and Consumer Affairs, Annual Report on Zoonoses in Denmark 2005, 2006, Copenhagen.
}

\section{MATERIALS AND METHODS}

\subsection{The Danish Swine Salmonellosis Control Programme}

The D.S.S.C.P. is based on the random testing of post-slaughter meat-juice samples from all finisher pig-herds that have an annual kill of greater than 200 finishers. The number of animals sampled at slaughter depends on herd size, with 60,75 , or 100 pigs sampled per herd per year [1]. The testing of meat-juice rather than blood facilitates both sample collection and carcass identification [24]. All samples are analysed at the Danish Institute for Food and Veterinary Research using the Danish mix-ELISA [23]. This test can detect O-antigens from at least $93 \%$ of all serovars that are known to be present in Danish pigs [21].

The sample results are used to categorise swine herds into one of three levels of a "serological Salmonella finisher index" [1]. The index for each herd was calculated using a three step process: (1) totalling the monthly number of positive samples (optical density greater than 20) for the last three months; (2) weighting the months' totals by $3: 1: 1$ so the most recent month carries the most weight; and (3) adding the weighted totals which are then divided by five. The three levels are "level 1" with an index of 1-39; "level 2" with an index of 40-69; and "level 3" with an index of 70 or more. Herds in levels 2 and 3 have requirements placed upon them e.g. pen faecal samples must be collected from the herd, producers must report their most recent weaner suppliers, and there are penalty "Salmonella deductions" resulting in reduced payments to these producers. Furthermore, pigs from level 3 herds are subject to special slaughter conditions. At the end of 2004, 3.5\% of finisher herds were assigned to level 2 and $1.1 \%$ to level $3^{1}$.

There have been a number of changes to the D.S.S.C.P. since its inception. These include a change in the sampling strategy in August 2001 [1] which was introduced to give more precise estimates for sero-prevalence in smaller herds.

\subsection{The data}

Two extracts of data were obtained from the central database of the D.S.S.C.P. from 1 January 1995 until 31 December 2004 (inclusive). The first data extract provided farm level information and included a unique identifier and the identification of the county and commune in which the farm was located. There are 275 communes within 14 larger 
counties in Denmark. The unique identifier is attached to the physical locality, and not the owner, thus ensuring correct identification of the farm over the ten-year study period. Due to the near complete coverage of sampling in the D.S.S.C.P. (all herds producing more than 100 finishers per annum prior to 1 August 2001, all producing more than 200 at or after 1 August 2001 to date), our data set was effectively a census comprising $99 \%$ of the population of Danish finisher swine herds.

Details of location of the farm house were provided for all farms that were registered with the Danish Central Husbandry Registry in March 2004. Amongst the 22344 farms sampled between 1 January 1995 and 31 December 2004, which had individual pig and farm level data, 14319 had recorded easting and northing coordinates. The remaining 8025 farms had coordinates randomly generated within their respective communes. This was an appropriate means for dealing with this type of missing data for two reasons. The size of those communes that produce pigs are small (range 20 to $58 \mathrm{~km}^{2}$ ) relative to the entire land area of Denmark $\left(43000 \mathrm{~km}^{2}\right)$, and inferences drawn from this study were made at a broad national level rather than at the small commune level. Furthermore, the need to randomly generate coordinates was time dependent: in 1995 this was necessary for 5940 of 16095 farms (37\%); in 1998, 4313 of 15790 farms (27\%); in 2001, 1204 of 11977 farms (10\%) and in 2004, only 173 of 9813 farms (2\%).

The second data extract provided information relating to the 6.8 million individual carcasses that were tested (out of a total kill of approximately 200 million) and included the date of sampling, the unique farm identifier, and the result of the Danish mix-ELISA. For this analysis, a result of greater than 20 optical density (O.D.\%) was classified as positive: this is the cut-off for positivity that has been used by the D.S.S.C.P. since 1 August 2001 [1]. The individual test sensitivity and specificity of the Danish mix-ELISA at this cut-off using meat juice has been estimated at $60 \%$ and $100 \%$ respectively $^{3}$.

${ }^{3}$ Enoe C., Andersen S., Wachmann H., Sorensen L.L., Estimation of sensitivity and specificity of an indirect enzyme-linked immunosorbent assay (ELISA) for detection of antibodies against Salmonella enterica in meat juice, in: Proceedings of the Fourth International Symposium on the Epidemiology and Control of Salmonella and other Foodborne Pathogens in Pork, Leipzig, 2001.

\subsection{Statistical analyses}

\subsubsection{Summary statistics}

We provided summary statistics at the county level for the ten year study period to determine a broad pattern of the variation in spatial risk. For each of the 14 counties (see Fig. 1) the number of farms and the proportion of samples yielding positive serology results were determined. The unit of analysis was the individual animal and confidence intervals for the proportion of positive serology results were calculated taking into account the presence of clustering at the farm level [11]. The proportion of positive serology results for a county was expressed as the county-level incidence risk (I.R.). For each county the O.D.\% values of positive serology results were aggregated and the lower, middle and upper quartiles calculated.

\subsubsection{Spatial analyses}

The land area of Denmark is comprised of a large peninsula (Jutland), two main islands (Fyn and Zealand), and 441 smaller islands (Fig. 1). Southern Jutland forms a border with northern Germany. Since the start of the D.S.S.C.P. over $85 \%$ of slaughter pigs have originated from the Jutland peninsula and Fyn.

To visualise the broad scale variability in farm density we calculated edge-corrected Gaussian kernel estimations [12] of the intensity function of the farm locations for each year of the study period. A fixed bandwidth was chosen for each year determined using the Gaussian optimal method [8]. To visualise broad-scale variability in sampled pig density we produced edge-corrected Gaussian kernel estimations of the intensity function of the count of sampled pigs by weighting the point locations of farms by the count of sampled pigs [2]. Since we did not have access to details of the number of pigs on each farm, we used the number of sampled pigs as a proxy for the farm-level pig population.

An important concept when describing spatial variation in risk is to adjust for the underlying population structure. The spatial relative risk function, estimated by kernel density methods, was developed for case control data for rare diseases by Bithell in 1990 [6]. Lawson and Williams (1993) and Kelsall and Diggle (1995) further improved the estimation $[16,17]$. In our analysis, we depart from the classic "case control" design since we have the unusual situation of what is effectively a census of pig farms. That is, we were not constrained by a strategy that required sampling of cases and 


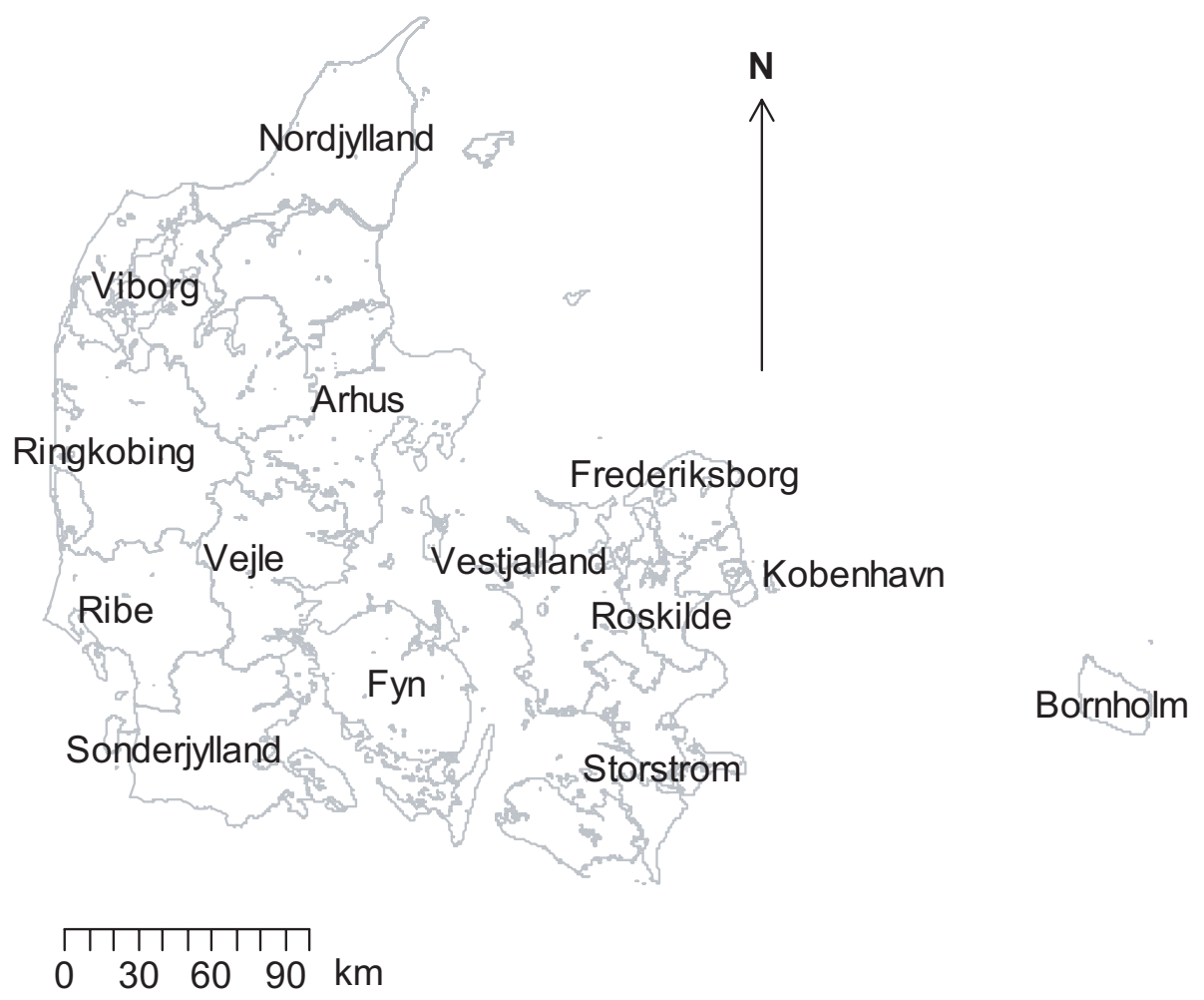

Figure 1. Map of Denmark showing the location of counties. The central island group containing Vestjalland, Roskilde, Storstrøm, Københaun and Fredriksborg is Zealand. The largest land mass containing Nordjylland, Viborg, Århus, Ringkobing, Vejle, Ribe and Sonderjylland is the Jutland peninsula. Southern Sonderjylland forms a border with northern Germany.

controls. This means we can directly estimate the intensity of disease and use the following function, $p(x)$ to estimate the conditional probability of a farm being a case at location $x$ :

$$
p(x)=\frac{\lambda_{1}(x)}{\lambda_{0}(x)+\lambda_{1}(x)}
$$

Equation (1) represents the probability of a farm being a case, conditional on its location. For each year of the study period farms were defined as cases if the proportion of sampled pigs that were positive was greater than or equal to 0.40 , otherwise farms were defined as non-cases. We chose this cut-off since it is the cut-off between levels 1 and 2 of the serological Salmonella finisher index. The function $\lambda_{1}(x)$ is the intensity of cases at an arbitrary point $x$, and $\lambda_{0}(x)$ is the intensity of non-cases at an arbitrary point $x$.
We obtained plug-in estimators of $\lambda_{1}$ and $\lambda_{0}$ using kernel smoothing:

$$
\begin{gathered}
\hat{\lambda}_{0}(x ; h)=\sum_{i=1}^{n_{0}} K_{h}\left(x-x_{i}\right) \\
\hat{\lambda}_{1}(x ; h)=\sum_{i=n_{0}+1}^{n} K_{h}\left(x-x_{i}\right)
\end{gathered}
$$

where $x_{1}, \ldots, x_{n_{0}}$ are the locations of the non-case farms, and $x_{n_{0}+1}, \ldots, x_{n}$ are the locations of the case farms. Also, $K_{h}(x)=h^{-2} K\left(h^{-1} x\right)$ with $K(\cdot)$ being a radially symmetric kernel function, and $h$ a smoothing parameter called the "bandwidth". The performance of the kernel density estimator depends on the choice of $h$. Fixed bandwidth kernel density estimators, as in Equation (2), have a major limitation when there is large spatial variation in the density of the population (as there is in the distribution of farms in Denmark). This results in an 
over-smoothing in areas of high density that could mask clusters of disease, and under-smoothing in areas of low density which could result in artificial spikes of disease risk.

We addressed this limitation by estimating conditional probability surfaces of a farm being a case for each year of the study period using a method for bandwidth selection that implements spatially adaptive smoothing. Specifically, we used sample point dependent bandwidths in Equation (2), so that for the $i$ th term in summation the bandwidth becomes $h_{i}=h\left(x_{i}\right)$. This bandwidth can be conveniently decomposed as $h_{i}=h \gamma_{i}$ where $\gamma_{i}$ is a local bandwidth factor (constrained to have a geometric mean of one over the samples of cases and non-cases) and $h$ a global smoothing multiplier common to both $\hat{\lambda}_{0}$ and $\hat{\lambda}_{1}$.

We selected the local bandwidth factors and global multiplier using a novel generalisation of the methodology of Hazelton [15], extended from the case of one dimensional $x$ values to two dimensional geographical locations. Specifically, we computed the local bandwidth factors by $\gamma_{i}=$ $g_{0} / \sqrt{\tilde{\lambda}_{0}\left(x_{i}\right)}$ and $\gamma_{i}=g_{1} / \sqrt{\tilde{\lambda}_{1}\left(x_{i}\right)}$ for non-cases and cases respectively, where $\tilde{\lambda}_{0}$ and $\tilde{\lambda}_{1}$ are pilot estimates of the respective intensity functions (constrained to be equal at coastal boundaries), and $g_{0}$ and $g_{1}$ are constants chosen to fix the geometric means of the bandwidth factors as described above. As a consequence, the local bandwidth factors are selected so as to apply the appropriate degree of smoothing in a relative sense (i.e. that which minimises mean square error), so that regions with a high intensity $\lambda$ of farms have smaller bandwidths than regions with low intensity. This means that data rich areas of fine detail (e.g. Viborg and Nordjylland) receive (far) less smoothing than geographical regions where the data are sparse (e.g. on Zealand). Meanwhile the global smoothing multiplier is adjusted to optimise the conditional probability surface as a whole (in terms of minimising an estimate of mean squared error, integrated over the geographical region), rather than the individual intensity functions per se.

Another important consequence of this methodology is that the estimated conditional probability surfaces are consistent at the boundary without any additional correction for bias introduced by edge effects. This boundary bias in a kernel comprises contributions from both the numerator (intensity of case farms at an arbitrary point) and denominator (intensity of all farms at an arbitrary point) of the conditional probability function. However, the use of the global smoothing multiplier leads to the cancellation of the leading bias terms from both numerator and denominator, resulting in a great reduction in the magnitude of the bias for the conditional probability function itself.

Inspection of maps of slaughter pig farm densities (Fig. 2) and the conditional probability surfaces (Fig. 3) provided a visual guide as to possible association between farm density and the risk of disease.

\section{RESULTS}

\subsection{Summary statistics}

The results from the descriptive analysis for counties are shown in Table I. There was a large variation in the ten year county-level I.R. The counties at the extreme north (Nordjylland) and south (Sonderjylland) of Jutland had the highest I.R. at $10 \%$ with very little uncertainty surrounding these estimates. The next three counties with 9\% I.R. were Fyn, Århus, and Viborg. The counties with the highest I.R. also demonstrated higher O.D.\% values for their lower, middle and upper quartiles of positive serology results. Counties on the islands of Zealand and Bornholm had $4 \%$ or less I.R., with the exception of Vestsjalland which had $6 \%$.

\subsection{Spatial analysis}

Edge-corrected Gaussian kernel estimations of the intensity functions of the farm locations and of the count of sampled pigs for each year of the study period were mapped. Slaughter pig farm density (Fig. 2) showed large variability across the country (from zero farms per square kilometer in Københaun to 0.6 in western Viborg in 1995). For presentation purposes Bornholm is excluded from the Figures. Farm density on Bornholm was similar to that on Fyn. The solid and dashed contour lines delineate the upper fifth and twentyfifth percentiles of farm density respectively. There was a reasonably uniform decrease in farm density over the ten year study period with the relative densities remaining very similar. For example even though farm density in western Viborg reduced from 0.6 to 0.4 farms per square kilometre over the ten year period, it remained the region with the highest farm density. Both farm (Fig. 2) and pig density (not 

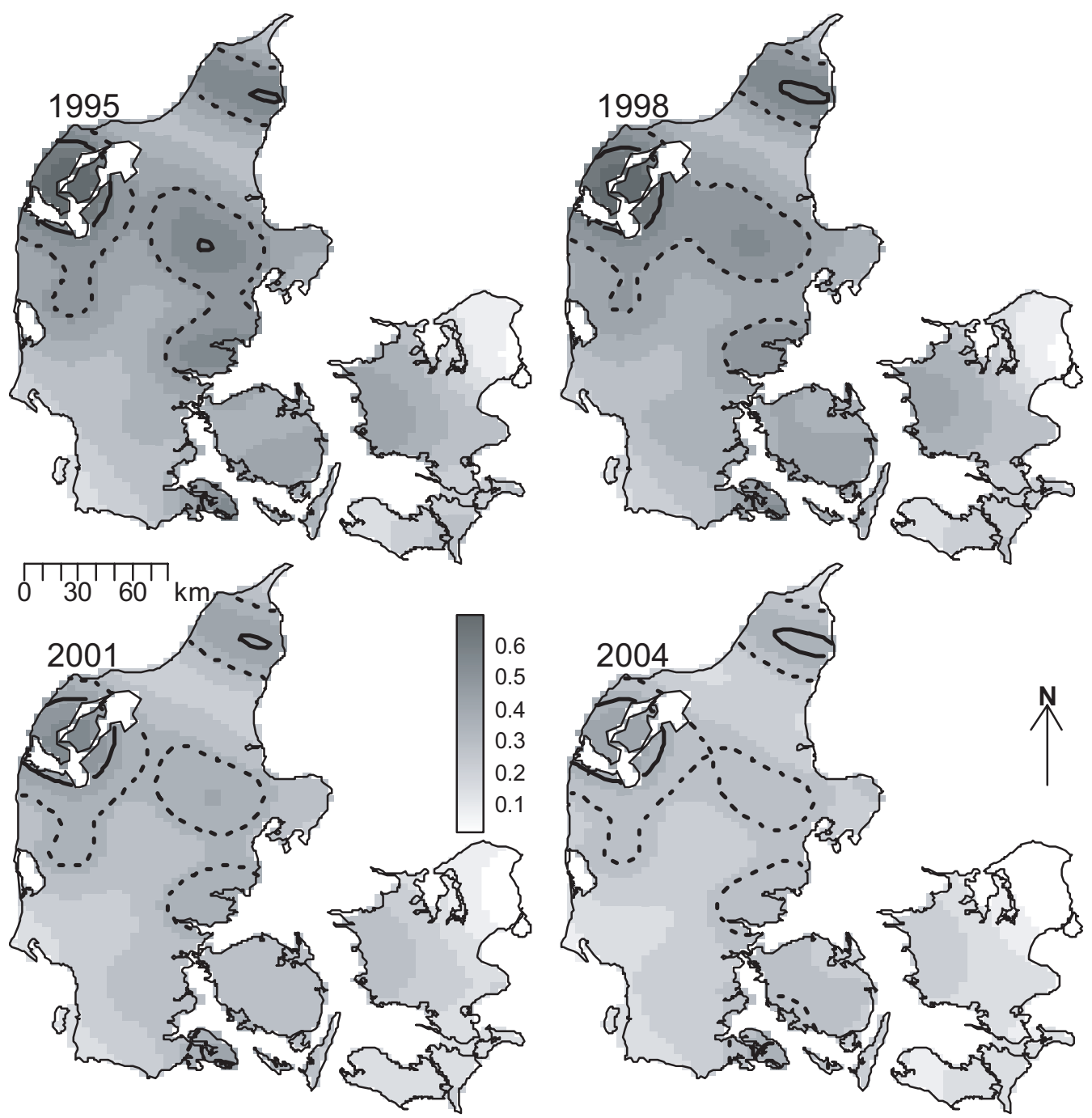

Figure 2. Edge-corrected kernel smoothed maps showing Salmonella meat-juice tested slaughter herd densities across Denmark in 1995, 1998, 2001 and 2004. The solid and dashed contour lines delineate the upper fifth and upper twenty-fifth percentiles of herd densities respectively. A fixed bandwidth of between 15.8 (1995) and $17.2 \mathrm{~km}(2004)$ was used.

shown) was the highest in northern Nordjylland, western Viborg, central Århus, eastern Vejle and south-eastern Sonderjylland (specifically on the island of Als). Throughout the whole study period, this farm and pig density pattern was reasonably consistent.

Figure 3 presents density plots of the conditional probability of a farm being a case for four years of the study period. The solid con- tour lines delineate the upper fifth percentile of Salmonella risk clearly showing areas where the conditional probability of a farm being a case is high. The dashed contour lines delineate the upper twenty-fifth percentile of risk. The variable bandwidths ranged between 4.4 and $62.5 \mathrm{~km}$. The geometric means of the bandwidths were between 8.6 and $12.6 \mathrm{~km}$. These maps show two spatial trends that are 

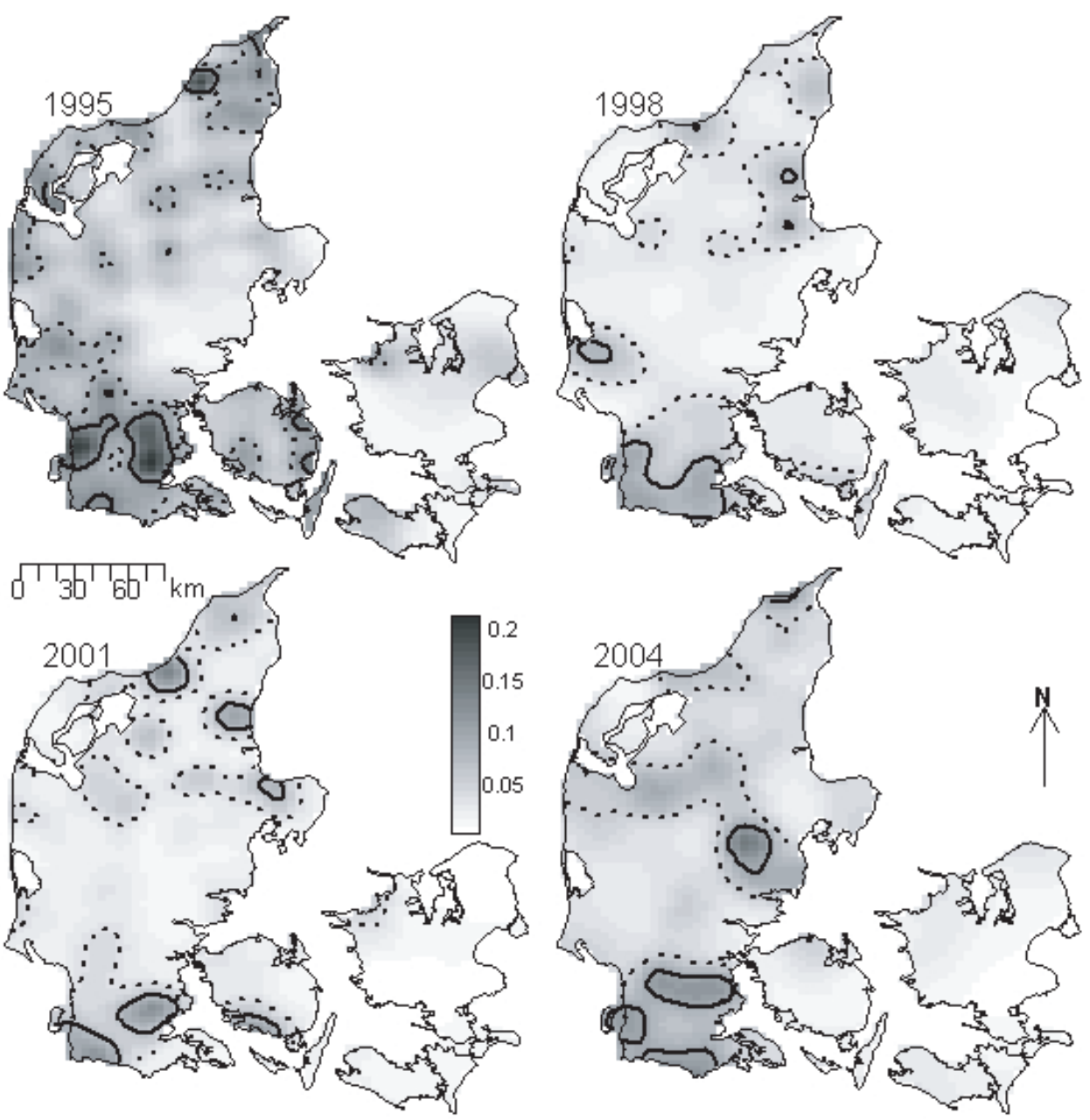

Figure 3. Salmonella seropositivity in Danish finisher pigs in 1995, 1998, 2001 and 2004. Density plots showing the conditional probability of a farm being a case. The solid and dashed contour lines delineate the upper fifth and upper twenty-fifth percentiles of Salmonella risk respectively.

consistent throughout the whole study period: (1) over the whole country there is increased conditional probability of a farm being a case on Jutland and Fyn when compared to Zealand and Bornholm; and (2) on the Jutland peninsula high conditional probabilities are in the south (especially the south-west) and to a lesser extent in the north. In addition to the consistent spatial trends in the conditional probability of a farm being a case over the ten years, there is a further trend that becomes ap- parent from 1998 onwards. This is a shift south in the northern polarity of risk on Jutland, and by 2004 there were pockets of central Jutland that had become areas of increased risk.

There was also a temporal pattern, with highest risk of Salmonella at the beginning and end of the study period. Six percent (905 of 16095 ) and 4\% (399 of 9 813) of farms were cases in 1995 and 2004 respectively. Three percent of farms in both 1998 (404 of 15 790) and 2001 (303 of 11 977) were cases. Visual 
Table I. Salmonella seropositivity in Danish finisher pigs, 1995-2004. Descriptive results by county.

\begin{tabular}{|c|c|c|c|c|c|c|c|}
\hline \multirow{2}{*}{ County name } & \multicolumn{3}{|c|}{ Number of } & \multirow{2}{*}{$\begin{array}{c}\text { Percent positive } \\
(95 \% \mathrm{CI})^{3}\end{array}$} & \multicolumn{3}{|c|}{ Positive summary ${ }^{4}$} \\
\hline & Farms tested & Results $^{1}$ & Positive results $^{2}$ & & 25 th & 50th & 75th \\
\hline Københaun & 8 & 1719 & 57 & $3(2-5)$ & 24 & 32 & 40 \\
\hline Frederiksborg & 238 & 40254 & 1501 & $4(3-4)$ & 24 & 32 & 49 \\
\hline Roskilde & 255 & 68322 & 2638 & $4(3-5)$ & 24 & 33 & 54 \\
\hline Vestjalland & 1577 & 406690 & 23257 & $6(5-6)$ & 26 & 38 & 69 \\
\hline Storstrøm & 1176 & 335530 & 12459 & $4(4-4)$ & 24 & 33 & 53 \\
\hline Bornholm & 468 & 149040 & 4994 & $3(3-4)$ & 24 & 31 & 46 \\
\hline Fyn & 1874 & 644171 & 58723 & $9(8-9)$ & 27 & 41 & 75 \\
\hline Sonderjylland & 1830 & 659878 & 64759 & $10(9-10)$ & 28 & 44 & 80 \\
\hline Ribe & 1163 & 303373 & 24636 & $8(8-9)$ & 28 & 44 & 79 \\
\hline Vejle & 1724 & 545750 & 39164 & $7(7-7)$ & 26 & 48 & 69 \\
\hline Ringkobing & 2797 & 946542 & 74245 & $8(8-8)$ & 26 & 38 & 70 \\
\hline Århus & 2527 & 754141 & 67774 & $9(8-9)$ & 25 & 36 & 64 \\
\hline Viborg & 3278 & 919622 & 79121 & $9(8-9)$ & 26 & 38 & 69 \\
\hline Nordjylland & 3429 & 993813 & 103438 & $10(10-11)$ & 26 & 40 & 76 \\
\hline Total & 22344 & 6768845 & 556766 & $8(8-8)$ & & & \\
\hline
\end{tabular}

${ }^{1}$ Individual pig serology results.

${ }^{2}$ An O.D. $\%$ of greater than 20.

${ }^{3}$ Adjusted for farm-level clustering.

${ }^{4}$ Quartile range of all positive results.

appraisal of the map series suggests no association between areas of high farm (Fig. 2) or pig density and areas with increased risk of Salmonella (Fig. 3).

In addition to the broad spatial trends, all maps showed evidence of aggregation of case farms visible as dark "spots" of varying diameter on the probability surfaces in Figure 3. This was most obvious in 1995 when the number of cases was the highest $(n=909)$.

\section{DISCUSSION}

This study has used a novel spatially adaptive smoothing technique to identify spatial patterns of slaughter pig Salmonella risk throughout Denmark. We found large variation in both county- and farm-level risk throughout Denmark with increased risk in the south and north of Jutland. The spatio-temporal analysis carries this further with visual confirmation of this pattern over the whole ten year study period. It has become apparent from this study that farms are most at risk in south-west Jutland and that those in central Jutland experienced increased risk from 1998 onwards.
We found that the geographical area within which a farm is located influences the risk for Salmonella and although farm location is not something that can be altered, it is useful to be aware of the high risk areas. More intensive sampling and gathering of epidemiological data in these areas may provide clues to underlying aetiologies. Qualitative assessment of the risk surfaces showed that case farms tend to aggregate, especially in 1995 when they were more numerous. This suggests that the underlying process is naturally clustered pointing to the need to explicitly model this structure in later work. When we visually assessed the relationship between farm and pig density and the farm-level risk of being a Salmonella case, we saw no association between them.

The kernel estimation of spatial relative risk is a useful tool in epidemiology which has been previously constrained by instability in estimates in areas where data are sparse [10]. Our application of a method for bandwidth selection which implements spatially adaptive smoothing overcomes this constraint 
and has the added advantage of self-correcting for edge effects. When compared with estimates computed using a fixed bandwidth, we were able to demonstrate appreciable improvements in performance with correction of over-smoothing in areas of low density, and under-smoothing in areas of high density. We believe this technique is a valuable addition to the repertoire available to spatial epidemiologists.

Our findings at the county-level are in agreement with earlier work on data from the D.S.S.C.P. $[9,21]$. Counties in the north and south of Jutland (Nordjylland and Sonderjylland) experienced increased Salmonella risk. Counties with the highest animal-level I.R. also demonstrated higher O.D.\% values for the low, middle and upper quartiles of their positive serology results. One possible explanation for this is that differences in the serological response depend on the serovars involved and the time since infection [23]. It is also very likely that the infection pressure within a herd contributes to an individual pig's level of sero-positivity. The pattern of a low positive summary for counties in Zealand (with the exception of Vestjalland) and Bornholm (Tab. I) may be a result of either low herd-level infection pressure or infection with serovars resulting in low test sensitivity, or both.

Caution must be used when interpreting the results from aggregated data since it may be subject to ecological bias [5]. If a county has a high I.R. this will not necessarily mean that all farms within that county are so affected; this becomes apparent when we investigate the farm level data. Notwithstanding our use of different measures of risk for county (proportion of samples positive) and farm, (case or non-case with cut-off of $40 \%$ of samples positive), we can identify that it is only parts of the most-affected counties that have an increased conditional probability of being a case. Sonderjylland experienced a high county-level I.R. at $10 \%$ but it is only the south-west of the county which consistently had the highest conditional probability of a farm being a case.

Our findings must be considered in the light of changes that have occurred in both the
D.S.S.C.P. sampling strategy and the Danish swine population during the study period. A small part of the reason for the decrease in farm density throughout the study period was due to a change in the D.S.S.C.P. that occurred in August 2001 [1]. The cut-off for herds to be eligible for sampling was raised from 100 to 200 pigs killed per year resulting in fewer herds being sampled with those that remained eligible being larger. This potentially results in selection bias, the direction and magnitude of which is unknown. However, we are confident it is likely to be of little consequence as over the study period herd sizes were increasing. In fact Danish pig herds doubled in size: in 1995 the number of finishers produced per herd was 1757 compared to 3043 in $2004^{4}$.

However, the reduction in the total number of suppliers producing pigs for slaughter was the more important reason for this decrease in farm density over the study period. For example, the number producing more than 200 pigs annually was approximately 13200 in 1994 reducing to 7800 in $2004^{2}$.

Another major change implemented in the D.S.S.C.P. in August 2001 resulted in more samples being taken from smaller herds and less samples from larger herds. Again this potentially results in bias of unknown direction and magnitude since the relationship between herd size and Salmonella risk is not straightforward (we address this more fully later in the discussion). Overall, both D.S.S.C.P. changes instigated in August 2001 led to a 13\% reduction in the total number of samples taken [1]. These changes in the sampling strategy confound our results for sampled pig density, allowing only empirical comparisons to be drawn.

The spatial patterns of Salmonella risk reported here allow us to generate a number of hypotheses. The pattern was possibly due to risk factors acting on a broad spatial scale such as a common infected source of pigs, contaminated feed, or regional biosecurity practices. A Danish study of the latter identified southern Jutland as an area where all surveyed sites hired commercial transport to the abattoir [7].

\footnotetext{
${ }^{4}$ National Committee for Pig Production, Annual Report 2005, 2006, Copenhagen.
} 
Transportation can be a biosecurity risk if, for example, transport personnel are allowed onto the farm ${ }^{5}$.

The south of Jutland has been identified as a high risk area for post-weaning multi-systemic wasting syndrome from 2001 to 2003 [29] and porcine reproduction and respiratory syndrome virus. There are published reports of the links between these diseases and salmonellosis in pigs $[4,14,22,31]$. Concurrent infections may act systemically to lower a pig's overall resistance to Salmonella or cause disturbances in the normal gut flora leading to increased susceptibility for Salmonella colonisation.

Denmark enjoys relative geographic isolation when compared with other northern European countries: Fyn, Zealand and Bornholm are true islands, and only the south of Sonderjylland is connected to the European mainland. The boundary between south Jutland and northern Germany may play as an yet undetermined role in the consistent risk of sero-positivity seen in the south-west of Sonderjylland. Although there is no movement of pigs across the German border into Denmark there is movement of pig feed, since farmers in the south of Jutland purchase feed across the border when the price is lower there. We speculate that this could be a potential source of Salmonella for herds in south Jutland, but further studies would need to be done to elucidate this.

Other risk factors acting on this scale could include regional variations in management practices such as home-mixing of feed in grain-producing areas with corresponding less use of pelleted feed. It is generally agreed that the feeding of pelleted feed is a risk for salmonellosis on pig farms $[18,20]$.

The two counties with the highest countylevel I.R. (Nordjylland and Sonderjylland) have large pig populations and generally larger farms. The association between herd size and Salmonella risk is complex: some studies report a positive association $[9,21]$ others a

\footnotetext{
${ }^{5}$ Baum D.H., Harris D.L., Nielsen B., FedorkaCray P.J., Risk Factors Associated with Increased Seroprevalence of Salmonella in Finishing Swine, in: The 15th International Pig Veterinary Society Congress, Birmingham, England, 1998.
}

negative [28] or no association [19,26]. Larger herds may have facilities that, in themselves, are sparing on Salmonella risk, e.g. feeding of fermented liquid feed $[4,28]$ and improved biosecurity $[13,19]$.

Since this is a descriptive study, we set out to generate the above hypotheses rather than specifically address the factors that may explain the patterns observed. By identifying these spatial patterns, we have highlighted the south-west of Sonderjylland on the Jutland peninsula as an area for further investigation and targeted surveillance. Furthermore, our exploration of the data has identified that case farms tend to aggregate. A method for investigating this issue further would be to model the data, accounting for both the broad spatial trend and the local spatial correlation structure.

\section{REFERENCES}

[1] Alban L., Stege H., Dahl J., The new classification system for slaughter-pig herds in the Danish Salmonella surveillance-and-control program, Prev. Vet. Med. (2002) 53:133-146.

[2] Baddeley A., Turner R., Spatstat: an R package for analyzing spatial point patterns, J. Stat. Soft. (2005) $12: 1-42$.

[3] Baggesen D.L., Wegener H.C., Phage types of Salmonella enterica ssp. enterica serovar typhimurium isolated from production animals and humans in Denmark, Acta Vet. Scand. (1994) 35:349-354.

[4] Beloeil P., Fravalo P., Fablet C., Jolly J., Eveno E., Hascoet Y., Chauvin C., Salvat G., Madec F., Risk factors for Salmonella enterica subsp. enterica shedding by market-age pigs in French farrow-to-finish herds, Prev. Vet. Med. (2004) 63:103-120.

[5] Biggeri A., Bohning D., Lesaffre E., Viel J.F., Lawson A., Divino F., Frigessi A., Introduction to spatial models in ecological analysis, in: Lawson A., Biggeri A., Bohning D., Lesaffre E., Viel J.F., Bertollini R. (Eds.), Disease mapping and risk assessment for public health, John Wiley and Sons, Chichester, 1999, pp. 180-192.

[6] Bithell J.F., An application of density estimation to geographical epidemiology, Stat. Med. (1990) 9:691701.

[7] Boklund A., Alban L., Mortensen S., Houe H., Biosecurity in 116 Danish fattening swineherds: descriptive results and factor analysis, Prev. Vet. Med. (2004) 66:49-62. 
[8] Bowman A.W., Azzalini A., Applied Smoothing Techniques for Data Analysis, Oxford University Press, Oxford, 1997.

[9] Carstensen B., Christensen J., Herd size and seroprevalence of Salmonella enterica in Danish swine herds: a random-effects model for register data, Prev. Vet. Med. (1998) 34:191-203.

[10] Clark A.B., Lawson A.B., An evaluation of nonparametric relative risk estimators for disease maps, Comp. Stat. Data Anal. (2004) 47:63-78.

[11] Dargatz D.A., Hill G.W., Analysis of survey data, Prev. Vet. Med. (1996) 28:225-237.

[12] Diggle P.J., A Kernel method for smoothing point process data, Appl. Stat. (1985) 34:138-147.

[13] Funk J.A., Davies P.R., Gebreyes W., Risk factors associated with Salmonella enterica prevalence in three-site swine production systems in North Carolina, USA, Berl. Munch. Tierarztl. Wochenschr. (2001) 114:335-338.

[14] Ha Y., Jung K., Kim J., Choi C., Chae C., Outbreak of salmonellosis in pigs with postweaning multisystemic wasting syndrome, Vet. Rec. (2005) 156:583-584.

[15] Hazelton M.L., Bias reduction in kernel binary regression, Comp. Stat. Data Anal. (2007) 51:43934402 .

[16] Kelsall J.E., Diggle P.J., Kernel estimation of relative risk, Bernoulli (1995) 1:3-16.

[17] Lawson A., Williams F., Application of extraction mapping in environmental epidemiology, Stat. Med. (1993) 12:1249-1258.

[18] Leontides L.S., Grafanakis E., Genigeorgis C., Factors associated with the serological prevalence of Salmonella enterica in Greek finishing swineherds, Epidemiol. Infect. (2003) 131:599-606.

[19] Lo Fo Wong D., Dahl J., Stege H., van der Wolf P., Leontides L., von Altrock A., Thorberg B., Herd-level risk factors for subclinical Salmonella infection in European finishing-pig herds, Prev. Vet. Med. (2004) 62:253-266.

[20] Mikkelsen L., Naughton P., Hedemann M., Jensen B., Effects of physical properties of feed on microbial ecology and survival of Salmonella enterica serovar Typhimurium in the pig gastrointestinal tract, Appl. Environ. Microbiol. (2004) 70:3485-3492.

[21] Mousing J., Jensen P.T., Halgaard C., Bager F., Feld N., Nielsen B., Nielsen J.P., Bech-Nielsen S., Nation-wide Salmonella enterica surveillance and control in Danish slaughter swine herds, Prev. Vet. Med. (1997) 29:247-261.
[22] Murakami S., Ogawa A., Kinoshita T., Matsumoto A., Ito N., Nakane T., Occurrence of swine salmonellosis in postweaning multisystemic wasting syndrome (PMWS) affected pigs concurrently infected with porcine reproduction and respiratory syndrome virus (PRRSV), J. Vet. Med. Sci. (2006) 68:387-391.

[23] Nielsen B., Baggesen D., Bager F., Haugegaard J., Lind P., The serological response to Salmonella serovars typhimurium and infantis in experimentally infected pigs. The time course followed with an indirect anti-LPS ELISA and bacteriological examinations, Vet. Microbiol. (1995) 47:205-218.

[24] Nielsen B., Ekeroth L., Bager F., Lind P., Use of muscle fluid as a source of antibodies for serologic detection of Salmonella infection in slaughter pig herds, J. Vet. Diagn. Invest. (1998) 10:158-163.

[25] Nielsen B., Alban L., Stege H., Sorensen L., Mogelmose V., Bagger J., Dahl J., Baggesen D., A new Salmonella surveillance and control programme in Danish pig herds and slaughterhouses, Berl. Munch. Tierarztl. Wochenschr. (2001) 114:323-326.

[26] Stege H., Christensen J., Nielsen J.P., Willeberg P., Data-quality issues and alternative variablescreening methods in a questionnaire-based study on subclinical Salmonella enterica infection in Danish pig herds, Prev. Vet. Med. (2001) 48:35-54.

[27] Tukey J.W., Exploratory data analysis, AddisonWesley, Reading, USA, 1977.

[28] Van der Wolf P., Wolbers W., Elbers A., van der Heijden H., Koppen J., Hunneman W., van Schie F., Tielen M., Herd level husbandry factors associated with the serological Salmonella prevalence in finishing pig herds in The Netherlands, Vet. Microbiol. (2001) 78:205-219.

[29] Vigre H., Baekbo P., Jorsal S.E., Bille-Hansen V., Hassing A.G., Enoe C., Botner A., Spatial and temporal patterns of pig herds diagnosed with Postweaning Multisystemic Wasting Syndrome (PMWS) during the first two years of its occurrence in Denmark, Vet. Microbiol. (2005) 110:17-26.

[30] Wegener H.C., Baggesen D.L., Investigation of an outbreak of human salmonellosis caused by Salmonella enterica ssp. enterica serovar Infantis by use of pulsed field gel electrophoresis, Int. J. Food Microbiol. (1996) 32:125-131.

[31] Wills R.W., Gray J.T., Fedorka-Cray P.J., Yoon K., Ladely S., Zimmerman J.J., Synergism between porcine reproductive and respiratory syndrome virus (PRRSV) and Salmonella choleraesuis in swine, Vet. Microbiol. (2000) 71:177-192. 